\title{
Pneumococcal Carriage and Antibiotic Resistance in Children Younger than 5 Years in Nouna District, Burkina Faso
}

Boubacar Coulibaly, ${ }^{1}$ Ali Sié, ${ }^{1}$ Dramane Kiemde, ${ }^{1}$ Nestor Dembélé, ${ }^{1}$ Adama Compaore, ${ }^{1}$ Ousmane Dabo, ${ }^{1}$ Clarisse Dah, ${ }^{1}$ Lucienne Ouermi, ${ }^{1}$ Vicky Cevallos, ${ }^{2}$ Elodie Lebas, ${ }^{2}$ Jessica M. Brogdon, ${ }^{2}$ Jeremy D. Keenan, ${ }^{2,3}$ and Catherine E. Oldenburg ${ }^{2,3,4 *}$

${ }^{1}$ Centre de Recherche en Santé de Nouna, Nouna, Burkina Faso; ${ }^{2}$ Francis I Proctor Foundation, University of California, San Francisco, San Francisco, California; ${ }^{3}$ Department of Ophthalmology, University of California, San Francisco, San Francisco, California; ${ }^{4}$ Department of Epidemiology and Biostatistics, University of California, San Francisco, San Francisco, California

\begin{abstract}
Increasing antibiotic consumption has been shown to lead to increased antibiotic resistance selection. We evaluated the prevalence of antibiotic resistance in Streptococcus pneumoniae to commonly used antibiotic classes as well as correlations between resistance and antibiotic consumption at the individual and community levels in children aged 0-59 months in Nouna district, Burkina Faso. A population-based sample of 300 children aged 0-59 months was randomly selected from the most recent census in 18 communities in the Nouna Health and Demographic Surveillance Site. Caregivers were interviewed about children's recent antibiotic use, and a nasopharyngeal swab was collected from each child. Nasopharyngeal swabs were processed using standard microbiological methods to determine pneumococcal carriage and resistance. Community-level antibiotic consumption was determined by record review from primary healthcare facilities, which routinely collect prescription data for children aged 0-59 months. Streptococcus pneumoniae was isolated from 101 (35.7\%) nasopharyngeal samples. Among positive isolates, co-trimoxazole (75.6\%) and tetracycline $(69.3 \%)$ resistance was the most common, followed by oxacillin (26.7\%) and azithromycin (9.9\%). Recent antibiotic use was associated with decreased pneumococcal carriage (odds ratio $0.56,95 \% \mathrm{Cl}: 0.33-0.93$ ) at the individual level. There was no statistically significant relationship between antibiotic use and antibiotic resistance at the individual or community levels, although Cls were generally wide. The prevalence of antibiotic resistance to commonly used antibiotics was high in the study area. Expanding antimicrobial resistance surveillance in areas with little population-based data will be important for informing policy related to antibiotic use.
\end{abstract}

\section{INTRODUCTION}

Streptococcus pneumoniae colonizes the nasopharynx in children, potentially causing invasive disease. ${ }^{1}$ Although introduction of the pneumococcal conjugate vaccine (PCV) has reduced the prevalence of invasive disease and mortality related to pneumococcal infection in children, $S$. pneumoniae remains an important contributor to childhood mortality in sub-Saharan Africa. ${ }^{2}$ Macrolides and beta-lactams, both commonly used antibiotic classes for childhood infections in sub-Saharan Africa, are first-line treatments for pneumococcal infection. ${ }^{3}$ In many areas of sub-Saharan Africa, children with symptoms of infection are treated empirically rather than on the basis of laboratory diagnostics, often because of the lack of diagnostic laboratory facilities particularly in rural areas. ${ }^{4}$ Although the $\mathrm{WHO}$ has recommended tailoring antibiotic treatment based on local resistance patterns, in practice, this is often difficult because of lack of resources to strengthen laboratory capacity for antimicrobial resistance surveillance. ${ }^{5}$

Antibiotic use has been shown to select for antibiotic resistance at the community and individual levels. ${ }^{6-10}$ Randomized controlled trials of mass azithromycin distribution for trachoma control and child mortality have demonstrated an increase in macrolide resistance prevalence in communities receiving azithromycin compared with untreated communities and that the prevalence of resistance tends to decrease when there is no selective pressure. ${ }^{6,9-11}$ Antibiotic treatment at the individual level has also been shown to select for resistance and lead to expansion of the gut resistome..$^{8,12-14}$

\footnotetext{
${ }^{*}$ Address correspondence to Catherine Oldenburg, Francis I. Proctor Foundation, University of California, San Francisco, 513 Parnassus Ave., Box 0412, San Francisco, CA 94143. E-mail: catherine. oldenburg@ucsf.edu
}

We conducted a population-based study of nasopharyngeal S. pneumoniae carriage and antibiotic resistance to commonly used antibiotic classes in Burkina Faso. ${ }^{3}$ Here, we report prevalence of resistance to macrolides, penicillin, and co-trimoxazole, as well as correlations between resistance and antibiotic consumption at the individual and community levels.

\section{MATERIALS AND METHODS}

Study setting. Figure 1 provides an overview of the study's methodology. This study took place in 18 communities in the Nouna Health and Demographic Surveillance Site (HDSS) in Nouna district, Boucle du Mouhoun Region, Burkina Faso, in July $2017 .{ }^{15}$ The area is in the sub-Sahel and is predominantly rural and agrarian. Vaccination coverage in the study area is high, with more than $80 \%$ of children being fully immunized according to the Expanded Programme on Immunization program in 2014 (defined as having received Bacillus CalmetteGuérin (BCG); oral polio vaccine 1, 2, and 3; pentavalent 1, 2, and 3 ; and measles vaccine by 12 months of age).${ }^{16} \mathrm{~A} 13$-valent PCV was introduced in Burkina Faso in 2013. ${ }^{17}$ In 2018, PCV13 coverage in Boucle du Mouhoun was $98 \% .{ }^{18}$ The institutional review boards at the Centre de Recherche en Sante de Nouna (CRSN) in Burkina Faso and the University of California, San Francisco in the United States reviewed and approved this study. Verbal informed consent was obtained from the caregiver of each participant before participating in the study.

Caregiver survey. Before nasopharyngeal swabbing, the caregiver of each child completed a brief survey related to their recent healthcare and antibiotic use. Caregivers were asked if their child had received any antibiotics in the last 3 months. To facilitate recall and minimize misclassification, caregivers were shown pictures of common antibiotics available in the 


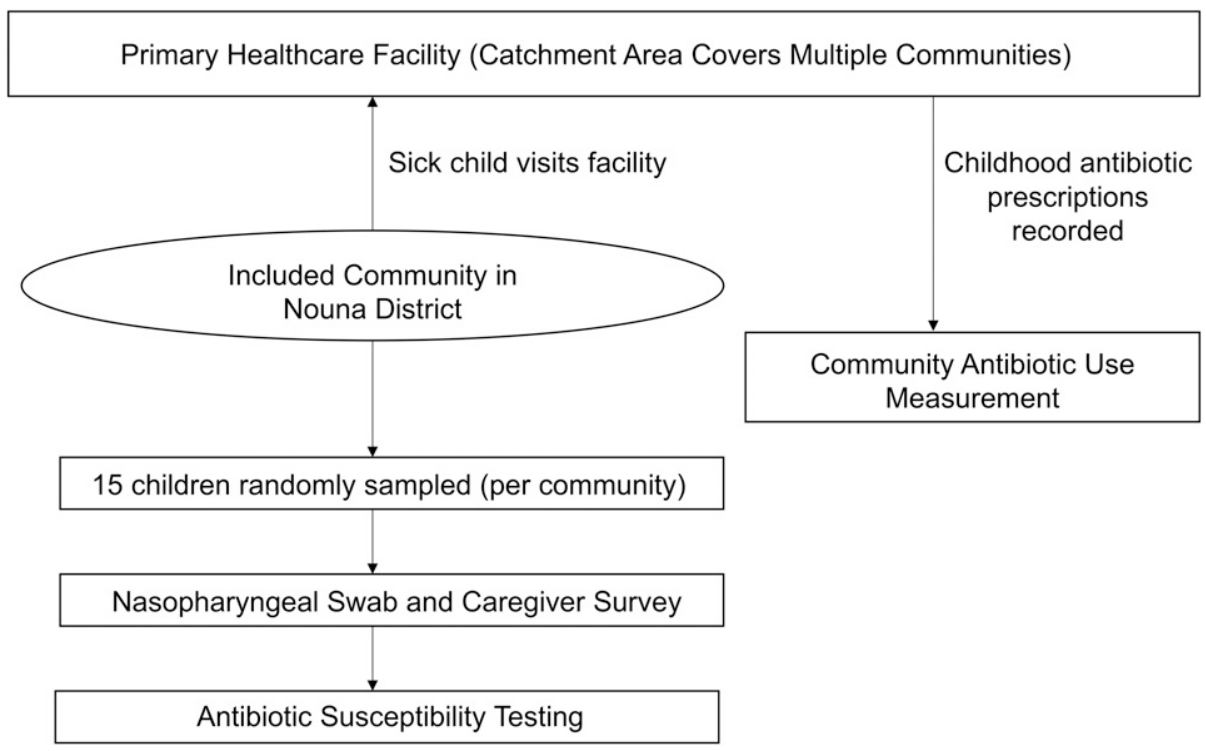

FIGURE 1. Study flowchart for study measurements and sources of data.

study area, as well as common antiparasitic and antimalarial medications. Caregivers were also asked if their child had recently been to the health center for a health problem and, if so, what the main reason for that visit was. Caregivers were allowed to report multiple reasons for the visit (e.g., fever and malaria).

Community antibiotic use measurement. Complete methods for the estimates of community antibiotic consumption have been previously published. ${ }^{3}$ In brief, we used records from primary healthcare facilities to count the number of antibiotic prescriptions in each study community. Health care is free for children younger than 5 years, and paper prescription slips are collected for each antibiotic prescription to obtain reimbursement from the Ministry of Health. We extracted antibiotic prescription data from six participating primary healthcare facilities as well as the child's age, gender, and village of residence. Rates of antibiotic prescription were calculated by dividing the number of antibiotic prescriptions by the total number of child-years contributed during the 3-month prescription period, estimated using census data from the HDSS.

Sample collection. In each study community, a random sample of 15 children aged 0-59 months were selected based on the most recent HDSS census. Four original study communities were combined into two because the delineation between the communities was arbitrary and not captured in primary healthcare facility antibiotic prescription records. Therefore, two study communities had 30 children randomly sampled. After providing verbal informed consent, the caregiver of each randomly selected child answered a brief questionnaire about the child's recent antibiotic use and any visits to the primary healthcare facility. A nasopharyngeal swab was collected from each child, placed in a cryotube containing skim milk-tryptone-glucose-glycerin (STGG) media on ice in the field, and then transported to the CRSN laboratory and stored at $-80^{\circ} \mathrm{C}$ until processing.

Laboratory methods. $0.1 \mathrm{~mL}$ of STGG from the cryotube containing the nasopharyngeal swab was plated and streaked on blood agar plates (Selective Strep Agar Base, CRITERION Dehydrated Culture Media, Hardy Diagnostics, Santa Maria,
CA). Streptococcus pneumoniae was identified on the selective media after incubation at $35^{\circ} \mathrm{C}$ in $5 \% \mathrm{CO}_{2}$ by Optochin disk testing (Fisher Scientific, Hanover Park, IL). Antimicrobial susceptibility testing was performed using Kirby-Bauer disc diffusion. Susceptibility testing was performed for azithromycin (15 ug), oxacillin (1 ug), tetracycline (30 ug), clindamycin (2 ug), and co-trimoxazole (23.75 ug/1.25 ug). Susceptibility was determined in accordance with the Clinical and Laboratory Standard Institute zone diameter interpretive standards for S. pneumoniae (2015 update). ${ }^{19}$ Intermediate and resistant isolates were determined to be non-susceptible. All laboratory tests were performed at the CRSN laboratory in Nouna, Burkina Faso.

Statistical methods. The prevalence of pneumococcal carriage and resistance to each antibiotic were calculated for the entire study population and by whether the child had recently received an antibiotic. We evaluated the relationship between recent antibiotic use and antibiotic resistance at the individual level using a generalized linear model with a Poisson distribution and robust standard errors to estimate risk ratios adjusted for the child's age and gender. At the community level, we calculated the prevalence of antibiotic resistance in each community and calculated correlation coefficients (rho) comparing resistance with antibiotic consumption expressed in prescriptions per child-year. All analyses were conducted in $R$ version 3.5.3 (The R Foundation for Statistical Computing).

\section{RESULTS}

Of 300 collected samples from 300 children, 283 (94\%) were successfully processed and linked to the caregiver antibiotic survey. The additional 17 samples were either missing in sample collection boxes or could not be linked to a child in the study. Of these, 111 (39.2\%) had received an antibiotic in the previous 3 months as per caregiver report. The distribution of age and gender was similar between children who had and had not received an antibiotic, although those who had received one were much more likely to have visited a health facility in the last month (Table 1). 
TABLE 1

Characteristics of included children

\begin{tabular}{lcc}
\hline & $\begin{array}{c}\text { Received an antibiotic, } \\
\text { past 3 months }(N=111)\end{array}$ & $\begin{array}{c}\text { Did not receive an antibiotic, } \\
\text { past 3 months }(N=172)\end{array}$ \\
\hline Age (months), median (IQR) & $34(21-46)$ & $40(26-50)$ \\
Female gender, $N(\%)$ & $53(47.7)$ & $91(52.9)$ \\
Health center visit, last 1 month, $N(\%)$ & $66(59.5)$ & $14(8.1)$ \\
Reason for health facility visit, $N(\%)$ & $56(50.5)$ & NA \\
Fever & $12(10.8)$ \\
Diarrhea & $66(59.5)$ \\
Malaria & $22(19.8)$ \\
Cough & $3(2.7)$ & \\
Other & $3.7)$ & \\
\hline
\end{tabular}

Streptococcus pneumoniae was isolated from 101 of the 283 children in the analytic sample (35.7\%). Antibiotic susceptibility testing was performed on all isolates for all antibiotics with the exception of oxacillin and co-trimoxazole, which were tested on $90(89 \%)$ S. pneumoniae isolates because of supply limitations. Among $S$. pneumoniae isolates, antibiotic resistance was highest to co-trimoxazole $(75.6 \%)$ and tetracycline $(69.3 \%)$, followed by oxacillin $(26.7 \%)$, azithromycin (9.9\%), and clindamycin (5.0\%) (Table 2). In models adjusted for the child's age and gender, recent receipt of an antibiotic was associated with significantly decreased pneumococcal carriage (odds ratio $0.56,95 \% \mathrm{Cl}$ : 0.33-0.93). There were no statistically significant associations between recent antibiotic use and resistance at the individual level (Table 2).

At the community level, there was no correlation between community antibiotic consumption (all classes) and pneumococcal carriage (rho $=0.006,95 \% \mathrm{Cl}$ : -0.46 to 0.47 ) (Figure 2A). Similarly, there was no evidence of correlation between amoxicillin consumption and prevalence of oxacillin resistance (rho $=0.23,95 \% \mathrm{Cl}$ : -0.28 to 0.64 ) (Figure $2 \mathrm{~B}$ ), erythromycin consumption and prevalence of azithromycin resistance (rho $=0.29,95 \% \mathrm{Cl}:-0.20$ to 0.67 ) (Figure $2 \mathrm{C}$ ), or co-trimoxazole consumption and prevalence of co-trimoxazole (rho $=0.1095 \% \mathrm{Cl}:-0.39$ to 0.54 ) (Figure 2D).

\section{DISCUSSION}

Resistance in S. pneumoniae to several commonly used antibiotics in the study area was high. Approximately $75 \%$ of isolates were resistant to co-trimoxazole, a finding that is similar to the prevalence of resistance in pneumococcal isolates from many settings in sub-Saharan Africa., ${ }^{90-23}$ Cotrimoxazole is commonly used for the treatment of pneumonia and prophylactically in children living with HIV or those who are HIV exposed but uninfected. ${ }^{24}$ The clinical implications of high levels of co-trimoxazole resistance are unclear, as some studies have shown that it remains effective despite high levels of resistance. ${ }^{25}$ Co-trimoxazole is the second most commonly prescribed antibiotic to children in the study area. ${ }^{3}$ At the community level, there was no evidence of a correlation between co-trimoxazole consumption and selection for cotrimoxazole resistance. At the individual level, the prevalence of co-trimoxazole resistance was higher in children recently treated with an antibiotic, but this did not reach statistical significance.

Amoxicillin is the most commonly prescribed antibiotic in the study area, ${ }^{3}$ and more than a quarter of isolates were resistant to oxacillin. Amoxicillin is also used prophylactically in children with uncomplicated severe acute malnutrition without clinical signs of infection due to the potential for asymptomatic infection due to suppressed immune systems in malnourished children. ${ }^{26-28}$ Mathematical modeling has indicated that pneumococcal resistance to beta-lactams may be responsible for treatment failures and increased morbidity and mortality in children in some settings. ${ }^{29}$ In areas similar to rural Burkina Faso, antibiotic exposure among young children is common, and most often antibiotics are administered in the absence of laboratory confirmation of infection or antimicrobial susceptibility testing. This may potentiate the overuse of antibiotics, for example, in viral infections or other scenarios in which they will be unhelpful. ${ }^{30}$

Pneumococcal carriage was lower in children with recent antibiotic exposure than in those without. The concentration of antibiotic achieved in tissues may be enough to reduce carriage even in individuals with resistant bacterial strains. ${ }^{31,32}$ Mass distribution of azithromycin for trachoma control leads to short-term reduction in pneumococcal carriage. ${ }^{6}$ In a study of seasonal malaria chemoprevention with or without azithromycin, pneumococcal carriage declined over time and was slightly lower in azithromycin-treated individuals at some time points. ${ }^{33}$ In the study area, children are vaccinated

TABLE 2

Pneumococcal carriage and phenotypic resistance in children aged 6-59 months, Nouna district, Burkina Faso

\begin{tabular}{|c|c|c|c|}
\hline & $\begin{array}{c}\text { Received an antibiotic, } \\
\text { past } 3 \text { months }(N=111), n(\%)\end{array}$ & $\begin{array}{l}\text { Did not receive an antibiotic, } \\
\text { past } 3 \text { months }(N=172), n(\%)\end{array}$ & Odds ratio $(95 \% \mathrm{Cl})$ \\
\hline Carriage & $32(28.8)$ & 69 (40.1) & $0.56(0.33-0.93)$ \\
\hline Azithromycin resistance* & $4(12.5)$ & $6(8.7)$ & $1.50(0.35-5.87)$ \\
\hline Oxacillin resistance* $\dagger$ & $10 / 29(34.4)$ & 14/61 (23.0) & $2.24(0.79-6.44)$ \\
\hline $\begin{array}{l}\text { Trimethoprim/sulfamethoxazole } \\
\text { resistance } \dagger\end{array}$ & $23 / 29$ (79.3) & 45/61 (73.8) & $1.67(0.57-5.39)$ \\
\hline Tetracycline resistance ${ }^{*}$ & $26(81.3)$ & $44(63.8)$ & $2.54(0.94-7.73)$ \\
\hline Clindamycin resistance* & $1(3.1)$ & $4(5.8)$ & $0.50(0.02-3.75)$ \\
\hline
\end{tabular}

*Analyses restricted to positive Streptococcus pneumoniae isolates.

†Oxacillin and trimethoprim/sulfamethoxazole resistance was not measured in all study communities; denominators are lower than the overall number of positive isolates. 

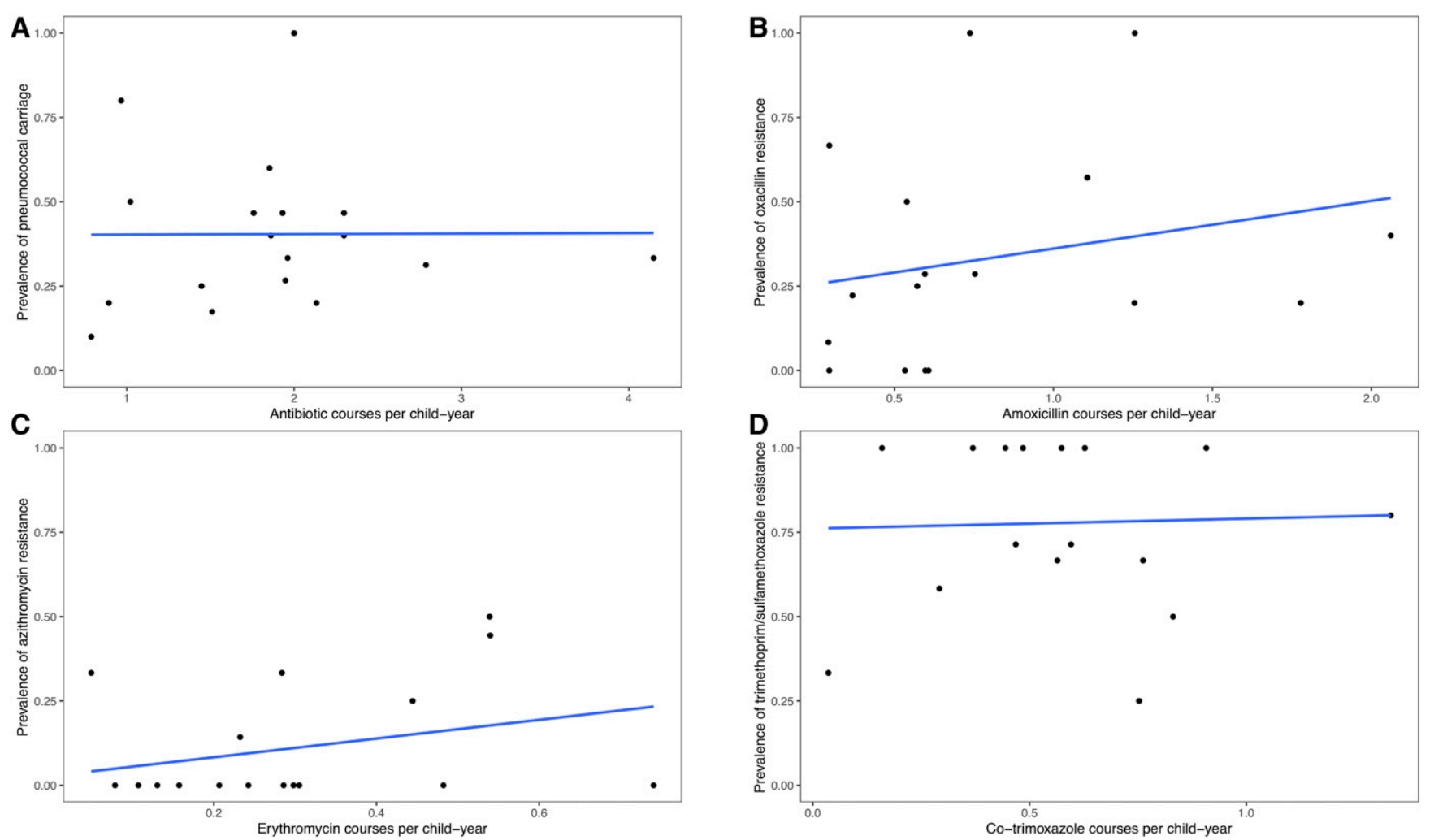

Figure 2. Community-level pediatric antibiotic consumption (all classes) and carriage of Streptococcus pneumoniae (A); community-level pediatric amoxicillin consumption and prevalence of oxacillin resistance (B); community-level pediatric erythromycin consumption and prevalence of azithromycin resistance (C); and community-level pediatric co-trimoxazole consumption and prevalence of co-trimoxazole resistance (D). Blue lines indicate linear trends for correlations between antibiotic consumption and carriage/resistance. This figure appears in color at www.ajtmh.org.

with 13-valent PCV. ${ }^{17}$ Although vaccination may reduce pneumococcal carriage, expansion of non-vaccine serotypes may occur following vaccination, filling the niche of the vaccine serotypes and resulting in continued high rates of colonization. ${ }^{34-37}$

There are several limitations to consider when interpreting these results. First, the sample size included was limited because of resource constraints, especially for community-level comparisons. We collected nasopharyngeal swabs from a population-based sample of children from 18 communities, meaning we had community-level estimates for only 18 communities. For individual-level associations, the sample size for resistance analyses was limited by the number of pneumococcal isolates. Although odds ratios were in the direction expected, Cls were wide, and we were unable to detect statistically significant associations between recent antibiotic exposure and antibiotic resistance in this setting. We are unable to comment based on these data as to whether there is a true relationship between routine antibiotic exposure and resistance selection. The caregiver survey did not elicit information about which class of antibiotics children received. Although we used pictorial tools to help ensure that caregivers accurately reported antibiotic receipt, we did not attempt to distinguish between specific antibiotic classes. Although cross-resistance is possible, comparison of resistance to specific antibiotic classes in children receiving those antibiotics may provide additional insights to these data. We did not serotype pneumococcal isolates. Although multiple serotypes can be resistant to antibiotics, the distribution of serotypes may be affected by vaccination with PCV. ${ }^{38}$ Finally, we did not collect data on PCV vaccination in communities or in children sampled. As previously discussed, PCV vaccination may affect carriage and resistance rates, which may vary by study community, and affect results of the study.

In this study, we found high prevalence of antibiotic resistance in S. pneumoniae to commonly used antibiotics in a rural area of Burkina Faso. Although the clinical implications of high background resistance is unclear, these results underscore the importance of expanding antimicrobial resistance surveillance in areas where few data currently exist, as well as better understanding the relationship between antibiotic consumption patterns and resistance. ${ }^{39,40}$

Received January 20, 2020. Accepted for publication May 2, 2020.

Published online May 26, 2020.

Financial support: C. E. O. was supported by a Research to Prevent Blindness Career Development Award.

Authors' addresses: Boubacar Coulibaly, Ali Sié, Dramane Kiemde, Nestor Dembélé, Adama Compaore, Ousmane Dabo, Clarisse Dah, and Lucienne Ouermi, Centre de Recherche en Sante de Nouna, Burkina Faso, E-mails: boubacar@fasonet.bf, sieali@yahoo.fr, kiemdedra@ gmail.com, sibirinestordembele@gmail.com, compaore.ada@outlook.fr, ousmanedabo15@yahoo.fr, n.clarissedah@yahoo.fr, and ouermil@ yahoo.fr. Vicky Cevallos, Elodie Lebas, Jessica M. Brogdon, and Catherine Oldenburg, Francis I Proctor Foundation, University of California, San Francisco, San Francisco, CA, E-mails: adavictoriacevallos@gmail. com, elodie.lebas@ucsf.edu, jessica.brogdon@ucsf.edu, and catherine. oldenburg@ucsf.edu. Jeremy D. Keenan, Francis I Proctor Foundation, Department of Ophthalmology, University of California, San Francisco, San Francisco, CA, E-mail: jeremy.keenan@ucsf.edu. 


\section{REFERENCES}

1. Simell B, Auranen K, Käyhty H, Goldblatt D, Dagan R, O'Brien KL; Pneumococcal Carriage Group, 2012. The fundamental link between pneumococcal carriage and disease. Expert Rev Vaccines 7: 841-855.

2. Wahl B et al., 2018. Burden of Streptococcus pneumoniae and Haemophilus influenzae type $b$ disease in children in the era of conjugate vaccines: global, regional, and national estimates for 2000-15. Lancet Glob Heal 6: e744-e757.

3. Sié A et al., 2019. Antibiotic prescription patterns among children younger than 5 years in Nouna district, Burkina Faso. Am J Trop Med Hyg 100: 1121-1124.

4. Lee ACC, Chandran A, Herbert HK, Kozuki N, Markell P, Shah R, Campbell H, Rudan I, Baqui AH, 2014. Treatment of infections in young infants in low- and middle-income countries: a systematic review and meta-analysis of frontline health worker diagnosis and antibiotic access. PLoS Med 11: e1001741.

5. Ayukekbong JA, Ntemgwa M, Atabe AN, 2017. The threat of antimicrobial resistance in developing countries: causes and control strategies. Antimicrob Resist Infect Control 6: 47.

6. O'Brien K, Emerson P, Hooper PJ, Reingold AL, Dennis EG, Keenan JD, Lietman TM, Oldenburg CE, 2018. Antimicrobial resistance following mass azithromycin distribution for trachoma: a systematic review. Lancet Infect Dis 19: e14-e25.

7. Lipsitch M, Samore MH, 2002. Antimicrobial use and antimicrobial resistance: a population perspective. Emerg Infect Dis 8: 347-354.

8. Oldenburg CE et al., 2020. Gut resistome after oral antibiotics in preschool children in Burkina Faso: a randomized controlled trial. Clin Infect Dis 70: 525-527.

9. Doan T et al., 2019. Macrolide resistance in MORDOR I-a clusterrandomized trial in Niger. N Engl J Med 380: 2271-2273.

10. Skalet AH et al., 2010. Antibiotic selection pressure and macrolide resistance in nasopharyngeal Streptococcus pneumoniae: a cluster-randomized clinical trial. PLoS Med 7: e1000377.

11. Haug $S$ et al., 2010. The decline of pneumococcal resistance after cessation of mass antibiotic distributions for trachoma. Clin Infect Dis 51: 571-574.

12. Chaw PS, Schlinkmann KM, Raupach-Rosin H, Karch A, Pletz MW, Huebner J, Nyan O, Mikolajczyk R, 2018. Antibiotic use on paediatric inpatients in a teaching hospital in the Gambia, a retrospective study. Antimicrob Resist Infect Control 7: 82.

13. Goossens H, Ferech M, Vander Stichele R, Elseviers M, 2005. Outpatient antibiotic use in Europe and association with resistance: a cross-national database study. Lancet 365: 579-587.

14. Costelloe C, Metcalfe C, Lovering A, Mant D, Hay AD, 2010. Effect of antibiotic prescribing in primary care on antimicrobial resistance in individual patients: systematic review and metaanalysis. BMJ 340: c2096.

15. Sie A, Louis VR, Gbangou A, Müller O, Niamba L, Stieglbauer G, Yé M, Kouyaté B, Sauerborn R, Becher H, 2010. The health and demographic surveillance system (HDSS) in Nouna, Burkina Faso, 1993-2007. Glob Health Action 3: 5284.

16. Kagoné M, Yé M, Nébié E, Sie A, Schoeps A, Becher H, Muller O, Fisker $A B, 2017$. Vaccination coverage and factors associated with adherence to the vaccination schedule in young children of a rural area in Burkina Faso. Glob Health Action 10: 1399749.

17. Kambiré $D$ et al., 2018. Early impact of 13-valent pneumococcal conjugate vaccine on pneumococcal meningitis - Burkina Faso, 2014-2015. J Infect 76: 270-279.

18. Ministere de la Sante Burkina Faso, 2019. Annuaire Statistique 2018. Ouagadougou, Burkina Faso.

19. Clinical and Laboratory Standards Institute, 2015. Performance Standards for Antimicrobial Disk Susceptibility Tests; Approved Standard, 12th edition. Wayne, PA.

20. Kobayashi $M$ et al., 2017. Pneumococcal carriage and antibiotic susceptibility patterns from two cross- sectional colonization surveys among children aged $<5$ years prior to the introduction of 10-valent pneumococcal conjugate vaccine-Kenya, 2009-2010. BMC Infect Dis 17: 25.
21. Sampane-Donkor E, Badoe EV, Annan JA, Nil-Trebi N 2017. Colonisation of antibiotic resistant bacteria in a cohort of HIV infected children in Ghana. Pan Afr Med J 26: 60.

22. Manenzhe RI, Moodley C, Abdulgader SM, Lourens Robberts FJ, Zar HJ, Nicol MP, Dube FS, 2019. Nasopharyngeal carriage of pneumococci in an intensively sampled South African birth cohort study population and sampling. Front Microbiol 10: 610.

23. Haile AA, Gidebo DD, Ali MM, 2019. Colonization rate of Streptococcus pneumoniae, its associated factors and antimicrobial susceptibility pattern among children attending kindergarten school in Hawassa, southern Ethiopia. BMC Res Notes 12: 344.

24. Chintu $\mathrm{C}$ et al., 2004. Co-trimoxazole as prophylaxis against opportunistic infections in HIV-infected Zambian children (CHAP): a double-blind randomised placebo-controlled trial. Lancet 364: 1865-1871.

25. Awasthi $S$ et al.; ICMR-IndiaClen Pneumonia Project Group, 2008. Effectiveness of 3-day amoxycillin vs. 5-day cotrimoxazole in the treatment of non-severe pneumonia in children aged 2-59 months of age: a multi-centric open labeled trial. J Trop Pediatr 54: 305-309.

26. Isanaka $S$ et al., 2016. Routine amoxicillin for uncomplicated severe acute malnutrition in children. N Engl J Med 374: 444-453.

27. Trehan I, Goldbach HS, LaGrone LN, Meuli GJ, Wang RJ, Maleta $\mathrm{KM}$, Manary MJ, 2013. Antibiotics as part of the management of severe acute malnutrition. N Engl J Med 368: 425-435.

28. World Health Organization, 2010. Guideline: Updates on the Management of Severe Acute Malnutrition in Infants and Children. Geneva, Switzerland: WHO.

29. Chen H, Stringer A, Eguale T, Rao GG, Ozawa S, Carolina N, 2019. Impact of antibiotic resistance on treatment of pneumococcal disease in Ethiopia: an agent-based modeling simulation. $\mathrm{Am} \mathrm{J}$ Trop Med Hyg 101: 1042-1053.

30. Tadesse BT, Ashley EA, Ongarello S, Havumaki J, Wijegoonewardena M, González IJ, Dittrich S, 2017. Antimicrobial resistance in Africa: a systematic review. BMC Infect Dis 17: 616.

31. Greenwood D, 1981. In Vitro veritas? Antimicrobial susceptibility tests and their clinical relevance. J Infect Dis 144: 380-385.

32. Rex JH, Pfaller MA, 2002. Has antifungal susceptibility testing come of age? Clin Infect Dis 35: 982-989.

33. Hema-Ouangraoua $S$ et al., 2019. Impact of the addition of azithromycin to antimalarials used for seasonal malaria chemoprevention on antimicrobial resistance of Streptococcus pneumoniae. Trop Med Int Heal 24: 1442-1454.

34. Kalata NL, Nyazika TK, Swarthout TD, Everett D, French N, Heyderman RS, Gordon SB, Jambo KC, 2019. Pneumococcal pneumonia and carriage in Africa before and after introduction of pneumococcal conjugate vaccines, 2000-2019: protocol for systematic review. BMJ Open 9: e030981.

35. Dobay O, 2019. The complexity of serotype replacement of pneumococci. Hum Vaccin Immunother 15: 2725-2728.

36. Lourenço $\mathrm{J}$ et al., 2019. Determinants of high residual postPCV13 pneumococcal vaccine-type carriage in Blantyre, Malawi: a modelling study. BMC Med 17: 219.

37. Sime WT, Aseffa A, Woldeamanuel Y, Brovall S, Morfeldt E, Henriques-normark B, 2019. Serotype and molecular diversity of nasopharyngeal Streptococcus pneumoniae isolates from children before and after vaccination with the ten-valent pneumococcal conjugate vaccine (PCV10) in Ethiopia. BMC Infect Dis 19: 409.

38. Linares J, Ardanuy C, Pallares R, Fenoll A, 2010. Changes in antimicrobial resistance, serotypes and genotypes in Streptococcus pneumoniae over a 30-year period. Clin Microbiol Infect 16: 402-410.

39. Wernli D, Peter SJ, Harbarth S, Carroll SP, Laxminarayan R, Levrat N, Røttingen JA, Pittet D, 2017. Antimicrobial resistance: the complex challenge of measurement to inform policy and the public. PLoS Med 14: e1002378.

40. Varma JK et al., 2010. Africa Centres for Disease Control and Prevention's framework for antimicrobial resistance control in Africa. Afr J Lab Med 7: 830. 